\title{
Determination of energy absorption in different cellular auxetic structures
}

\author{
M. Shokri Rad ${ }^{1}$, Hossein Hatami ${ }^{1, *}$, R. Alipouri ${ }^{2}$, A. Farokhi Nejad ${ }^{3}$, and F. Omidinasab ${ }^{4}$ \\ ${ }^{1}$ Department of Mechanical Engineering, Lorestan University, Khorramabad, Lorestan, Iran \\ ${ }^{2}$ Department of Mechanical Engineering, Mahshahr Branch, Islamic Azad University, Mahshahr, Iran \\ ${ }^{3}$ Department of Mechanical and Aerospace Engineering, Politecnico di Torino, Turin, Italy \\ ${ }^{4}$ Department of Civil Engineering, Lorestan University, Khorramabad, Lorestan, Iran
}

Received: 20 May 2018 / Accepted: 15 March 2019

\begin{abstract}
This paper deals with the effect of unit cell configuration on the energy absorption response of different cellular auxetic structures subjected to quasi-static and dynamic loadings through the experimental and numerical methods. Among the various structures, a re-entrant structure was selected due to its fundamental properties underlying the main characteristics of an auxetic material. Computer simulation techniques using ABAQUS software validated by experimental testing were used to conduct the evaluation of such devices. Several re-entrant structures with different geometrical parameters were modeled and compared with the conventional ones. Standard compression tests were carried out on the different structures produced by the $3 \mathrm{D}$ printing machine to evaluate the influence of auxeticity phenomenon in the energy absorption capability. It is discovered that the auxetic structures are superior to non-auxetic structures in terms of all studied impact resistance and energy absorption indicators due to their ability to withstand quasi-static axial impact loads. The primary outcome of this research is to extract design information for the use of auxetic materials as energy absorbers where quasi-static loading is expected.
\end{abstract}

Keywords: Auxetic structures / energy absorption / finite element / 3D Printing / experimental method

\section{Introduction}

Nowadays, modern technology and industries require new materials of special properties. One of the reasons for interest in materials of unusual mechanical properties comes from the fact that they can be used as matrices to form composites with other materials of other required properties, e.g. electric, magnetic, etc. A new field of endeavor is to study materials exhibiting negative Poisson's ratio (NPR). These new types of materials have been named auxetic in which, in contrast to conventional materials (like rubber, glass, metals, etc.), transverse expansion causes longitudinal pull out and vice versa [1]. Some of the important mechanical properties such as indentation resistance, energy absorption, impact resistance, fracture toughness, fatigue toughness and shear modulus are mainly influenced by the NPR [2-6]. Therefore, the application of auxetic materials, as a new class of materials, in numerous industries such as

\footnotetext{
* e-mails: hatami.h@lu.ac.ir; h64hatami@gmail.com
}

aeronautical, automotive and crash safety conservation structures to dissipate and absorb the direct impact loads is a part of contribution $[7,8]$.

Due to the fact that most of the engineering materials have positive Poisson's ratio, it might be normally imagined that Poisson's ratio is always positive. However, it has been proven theoretically that Poisson's ratio for normal materials can have either positive or negative value, only if the strain energy is positive [9]. As the first experimental approach [10], Lakes suggested open-cell PVC foam for fabrication of auxetic materials. His approach was modified by Bianchi et al. [11] by optimizing the manufacturing process. Another fabrication method was presented by Grima et al. [12] who re-transferred auxetic specimens to conventional one by putting them in acetone and then drying in air. The mentioned methods have been modified, developed and followed by the other researchers in filed $[13,14]$. Heating time, heating temperature, cell size, cell shape, and volumetric compression ratio have been referred as the most effective parameters, in almost all reports contributed to the manufacturing process of auxetic foams [11,15-20]. Hence, the other 


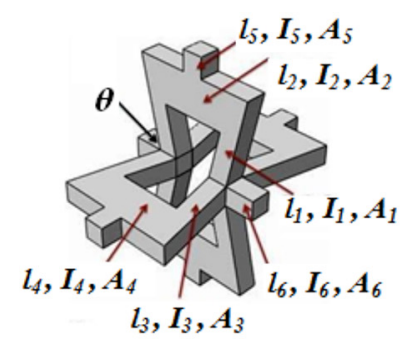

(a)

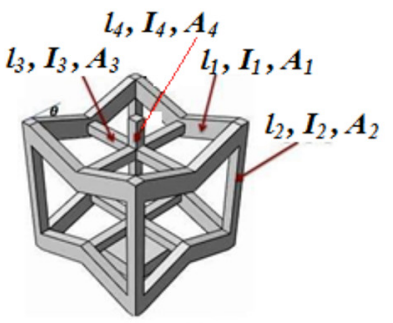

(c)

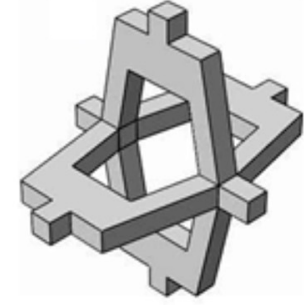

(b)

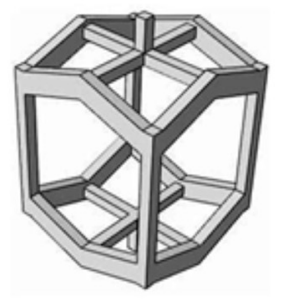

(d)

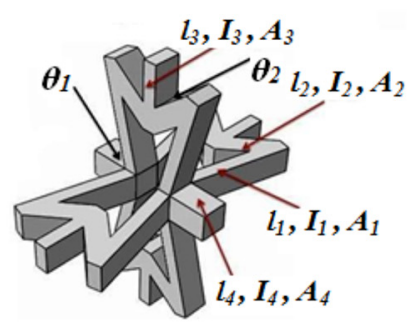

(e)
Fig. 1. Geometrical specification of unit cells.

researches in the term of auxeticity are more or less focused on the mentioned parameters to improve the fabrication process of auxetic materials [21-25].

Impact resistance and energy absorption of auxetic structures is also an attractive issue that researchers have been paying much attention to [26-28]. Reid and Peng [29] developed the one-dimensional shock theory evaluating the aspects of a crushing front through the wood subjected to the uniaxial impact. Ruan et al. [30] employed finite element analysis (FEA) to study the efficacy of impact speed and wall thickness of cell on the localized deformation state and plateau stress. The effect of cell-wall angles on the dynamic collision reaction of a re-entrant honeycomb structure was investigated by Zhang et al. [31]. It was realized that enhancing in the impact speed, cell angle and relative density leads to increasing the crashworthiness capability of the auxetic structure. A similar research on hexagonal cellular structure has been conducted by $\mathrm{Hu}$ and his colleagues [32], where it was shown that a honeycomb with a cell angle of $45^{\circ}$ has the best energy absorption performance subjected to the impact force. Three different deformation patterns under impact loading of cellular structures were discussed by Zou et al. [33]. Based on the collapsing mechanism of hexagonal honeycomb structures, an analytical formulation for the energy absorption ability was derived by $\mathrm{Hu}$ et al. [34]. They later developed an analytical model validated by the simulations to anticipate the crashworthiness of hexagonal honeycombs under low impact loading [35].
To exhibit the auxetic property, auxetic materials should possess substantial porosity in their microstructures [1]. So, geometric complexity and porosity in these structures under numerical study make their analysis cumbersome. In terms of finite element work, previous studies in this area show that numerical simulation of energy absorption of auxetic materials especially in threedimensional re-entrant structures is still limited and sparse, thus needing further development [36]. In the present study, finite element approach and experimental method are employed to calculate energy absorption and impact resistance of auxetic and conventional unit cells. After discovering the best energy absorber unit cells, they used to provide a cellular auxetic structure. These structures are modeled through the finite element approach to the energy absorption and impact resistance.

\section{Geometrical specifications of conventional and auxetic unit cells}

In this paper, several auxetic re-entrant cells with different shapes are investigated in the case of energy absorption application. An experimental method and finite element approach are employed for different geometrical specifications of the mentioned unit cells to obtain the energy absorption capability. Also, some conventional unit cells are modeled to compare their energy absorption capability with the auxetic unit cells. Figure 1 and Table 1 show the shapes and geometrical parameter values of the modeled unit cells, respectively.

\section{Experimental procedures}

Fabrication process and experimental procedure are explained in this section. The energy absorption of different unit cells under quasi-static loading is experimentally determined.

\subsection{Materials and fabrication process}

Acrylonitrile Butadiene Styrene (ABS) which is a common thermoplastic material was selected to make the unit cells due to availability and ease of production process. The most momentous specifications of ABS are toughness and impact resistance.

Different unit cells were first fabricated by using 3D printing machine. Figure 2 shows different shapes of the fabricated structures.

\subsection{Experiments}

Quasi-static compression test on the unit cells was done by using a SATEC universal testing machine as shown in Figure 3. Each experiment encompassed 5 samples and the average of the results was applied for the energy absorption evaluation. The rate of loading was adjusted to $1.5 \mathrm{~mm} / \mathrm{min}$.

Compressive load-compressive extension curves of the unit cells achieved from experiments were used to calculate the elasticity modulus and energy absorption capacity of 
Table 1. Geometrical parameter values of the unit cells.

\begin{tabular}{|c|c|c|c|c|}
\hline Code & Figure & number & Type of unit cell & Geometrical specifications (length $(\mathrm{mm})$ ) \\
\hline $\mathrm{A} 1$ & Figure & $1 \mathrm{a}$ & Auxetic & $\begin{array}{l}L_{1}=L_{3}=17.95, L_{2}=L_{4}=21, L_{5}=4.5, L_{6}=8.2 \\
A_{1}=A_{3}=A_{5}=A_{6}=31, A_{2}=A_{4}=47 \\
I_{1}=I_{3}=I_{5}=I_{6}=48.09, I_{2}=I_{4}=512.66 \\
\theta=75.4^{0}\end{array}$ \\
\hline $\mathrm{C} 1$ & Figure & $1 b$ & Conventional & $\begin{array}{l}L_{1}=L_{3}=17.95, L_{2}=L_{4}=21, L_{5}=4.5, L_{6}=8.2 \\
A_{1}=A_{3}=A_{5}=A_{6}=31, A_{2}=A_{4}=47 \\
I_{1}=I_{3}=I_{5}=I_{6}=48.09, I_{2}=I_{4}=386.63 \\
\theta=-75.4^{0}\end{array}$ \\
\hline $\mathrm{A} 2$ & Figure & $1 \mathrm{c}$ & Auxetic & $\begin{array}{l}L_{1}=15, L_{2}=42, L_{3}=15.28, L_{4}=6 \\
A_{1}=14.6, A_{2}=A_{3}=A_{4}=7.34 \\
I_{1}=27, I_{2}=I_{3}=I_{4}=4.37 \\
\theta=67.5^{0}\end{array}$ \\
\hline $\mathrm{C} 2$ & Figure & $1 d$ & Conventional & $\begin{array}{l}L_{1}=15, L_{2}=42, L_{3}=15.28, L_{4}=6 \\
A_{1}=14.6, A_{2}=A_{3}=A_{4}=7.34 \\
I_{1}=27, I_{2}=I_{3}=I_{4}=4.37 \\
\theta=-67.5^{0}\end{array}$ \\
\hline A3 & Figure & $1 \mathrm{e}$ & Auxetic & $\begin{array}{l}L_{1}=41.2, L_{2}=14, L_{3}=16.5, L_{4}=13.8 \\
A_{1}=A_{2}=A_{3}=48, A_{4}=96 \\
I_{1}=I_{2}=I_{3}=383.25, I_{4}=728.21 \\
\theta_{1}=72.8^{0}, \theta_{2}=47.1^{0}\end{array}$ \\
\hline A5 & Figure & $1 \mathrm{a}$ & Auxetic & $\begin{array}{l}L_{1}=16.7, L_{2}=24.14, L_{3}=25.32, L_{4}=24.45, L_{5}=7, L_{6}=7.27 \\
A_{1}=A_{2}=A_{3}=A_{4}=69, A_{5}=A_{6}=57 \\
I_{1}=I_{2}=I_{3}=I_{4}=216, I_{5}=I_{6}=113 \\
\theta=74.2^{0}\end{array}$ \\
\hline $\mathrm{A} 6$ & Figure & $1 \mathrm{c}$ & Auxetic & $\begin{array}{l}L_{1}=3.94, L_{2}=14, L_{3}=8.2, L_{4}=4.5 \\
A_{1}=14, A_{2}=A_{3}=A_{4}=24 \\
I_{1}=13.25, I_{2}=I_{3}=I_{4}=47.53 \\
\theta=42.1^{0}\end{array}$ \\
\hline A7 & Figure & $1 \mathrm{e}$ & Auxetic & $\begin{array}{l}L_{1}=38, L_{2}=16.21, L_{3}=13.8, L_{4}=12.67 \\
A_{1}=A_{3}=A_{4}=23.5, A_{2}=47 \\
I_{1}=I_{3}=I_{4}=54.71, I_{2}=463.5 \\
\theta_{1}=73.8^{0}, \theta_{2}=57.3^{0}\end{array}$ \\
\hline A 8 & Figure & $1 \mathrm{a}$ & Auxetic & $\begin{array}{l}L_{1}=17.27, L_{2}=15.83, L_{3}=33.4, L_{4}=36.25, L_{5}=7, L_{6}=7.5 \\
A_{1}=A_{2}=A_{3}=A_{4}=A_{5}=69, A_{6}=128 \\
I_{1}=I_{2}=I_{3}=I_{4}=I_{5}=231, I_{6}=1623 \\
\theta=63^{0}\end{array}$ \\
\hline
\end{tabular}

unit cells. An example of these curves is plotted in Figure 4 for unit cell A2.

Elastic modulus and energy absorption of each type of unit cells were calculated using the following equations [37]:

$$
E=\frac{\sigma}{\varepsilon}=\frac{P L}{A \delta},
$$

$$
U_{t}=\frac{1}{2} P \delta=\frac{P^{2} L}{2 A E},
$$

where, $P, L, A$ and $\delta$ are compression load, height of the unit cell, the area perpendicular to the loading direction and compression value of the structure, respectively. 

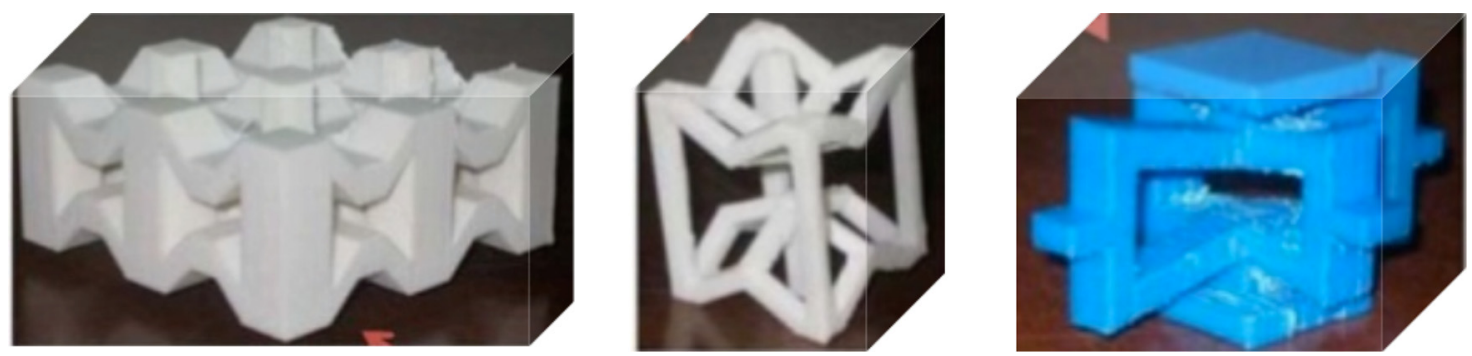

Fig. 2. Some of the structures fabricated by $3 \mathrm{D}$ printing machine.

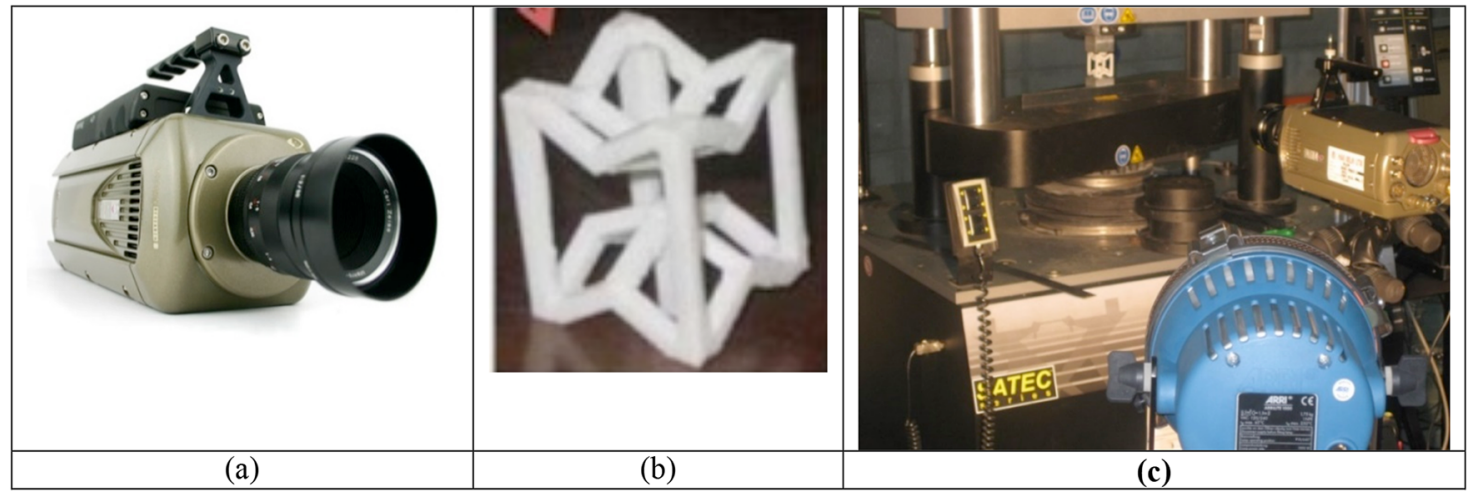

Fig. 3. a: High speed camera model phan. V710; b: 3D auxetic model; c: quasi-static compression test.

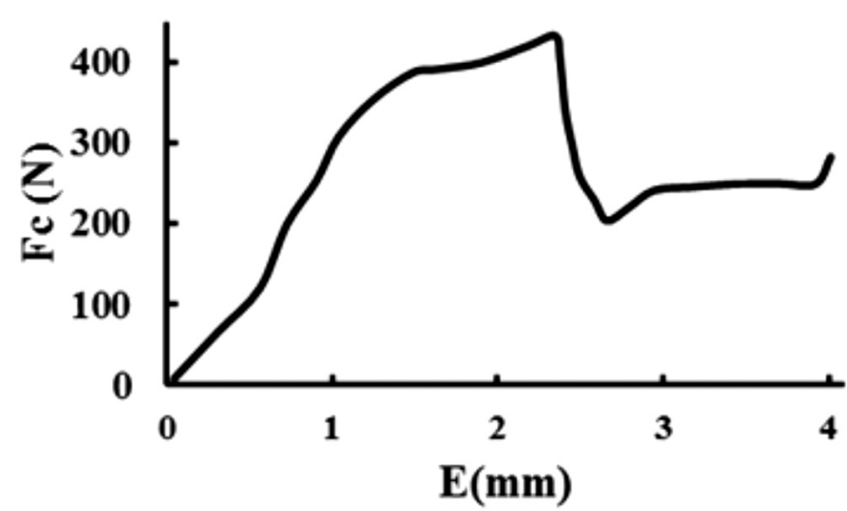

Fig. 4. Load-displacement curve of unit cell A2.

\section{Finite element simulations}

General conditions of numerical simulation are as follows. Computer simulations were carried out on the unit cells employing the nonlinear finite element (FE) software ABAQUS/Explicit. Models of the unit cells were meshed using hexahedral elements with 8 nodes (C3D8R). After convergence, element sizes of $0.5 \mathrm{~mm}$ for the unit cells were determined to obtain acceptable responses. The jaws of universal testing machine were modeled using a rigid body. Surface-to-surface contact was employed to define the contact condition between the jaws and the materials. The lower jaw was constant and the upper one moved downward with the speed of $1.5 \mathrm{~mm} / \mathrm{min}$ as same as

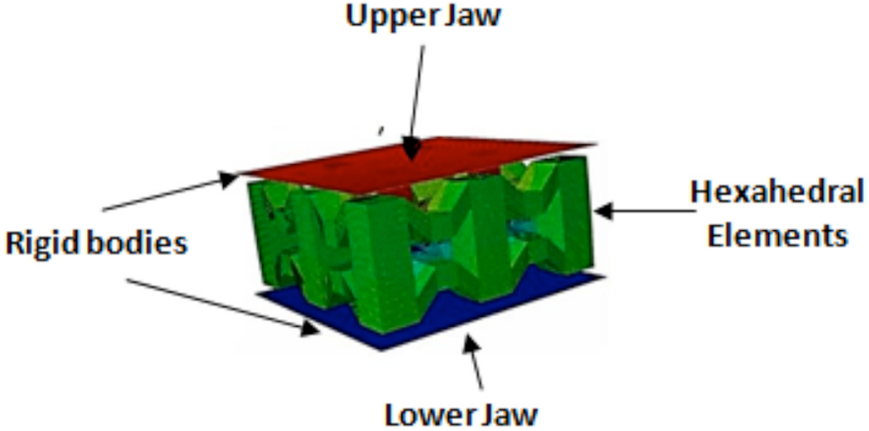

Fig. 5. A FE model of unit cells.

experiments. Similar boundary conditions to experiments were exerted to all FE simulations utilizing a couple of reference points i.e. the lower jaw was fixed in all degrees of freedom and the upper one was allowed to move along loading direction.

The FE study includes two parts: simulation of a unit cell and then extending the unit cell to an auxetic structure for investigation the energy absorption and impact resistance capabilities.

\subsection{Unit cell simulation}

Since the behavior of ABS is completely brittle, just the elastic part of its mechanical property was used to conduct the FE simulation. The isotropic elastic model was used to simulate the unit cells. Figure 5 shows a FE model of a unit cell sample. 


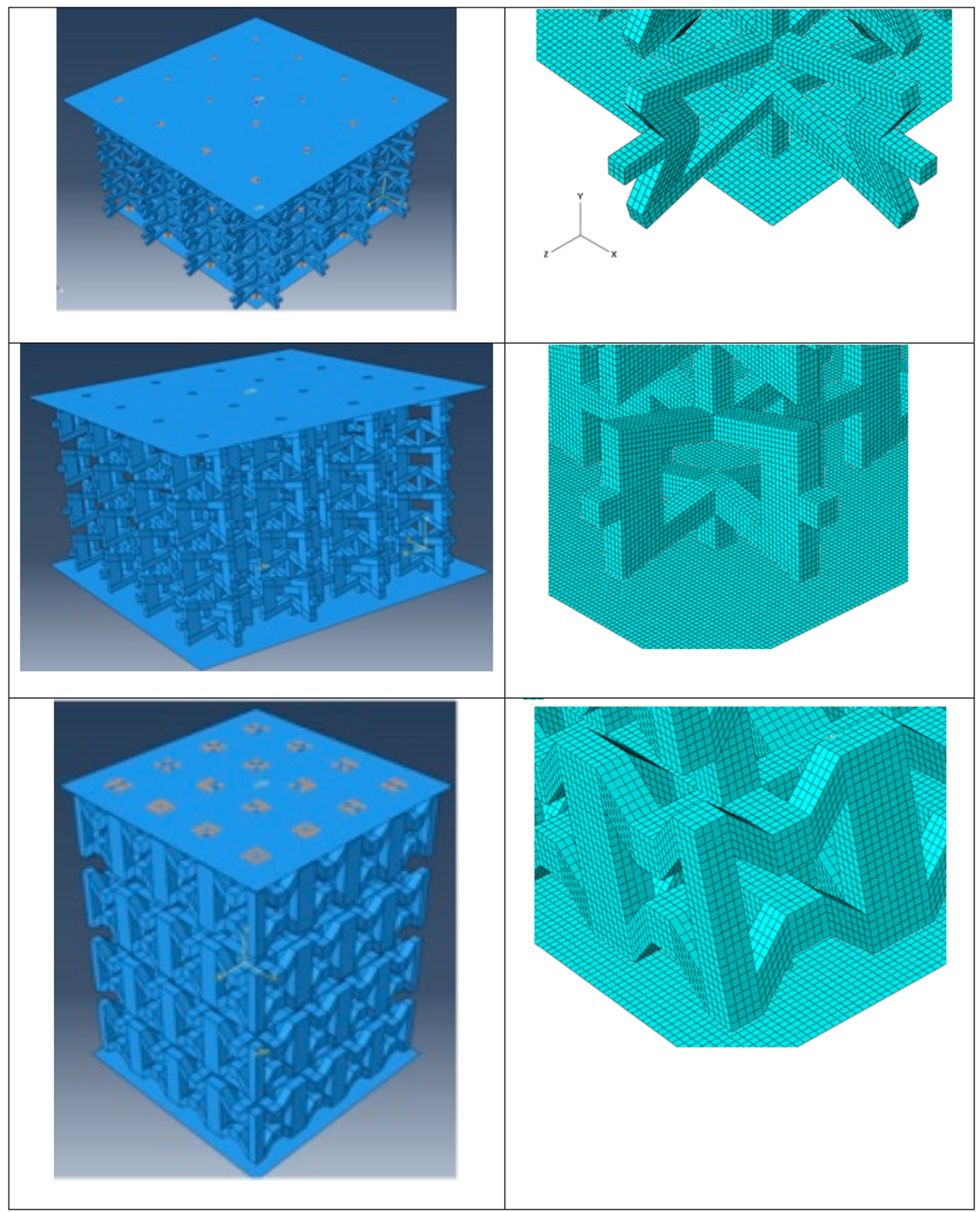

Fig. 6. Finite element models of different samples of cellular auxetic structures.

\subsection{Simulation of cellular auxetic structures}

Best auxetic unit cells based on their energy absorption capability were selected to extend for providing a cellular auxetic structure. The geometry of these structures is cubic consisting of 64 unit cells. Geometry parameters values of unit cells are shown in Table 1. Finite element models of different samples of cellular auxetic structures are shown in Figure 6 .
FE simulations were carried out for different cellular auxetic structures to obtain energy absorption capability. Mechanical properties of AISI 1052 steel were used for modeling the cellular auxetic structures. This material is ductile and thus plastic behavior observation of structures under loading is suitably possible. It is worthwhile to mention that using this material for modeling is only for comparative study of energy absorption capability of different geometries. The isotropic plasticity model was 


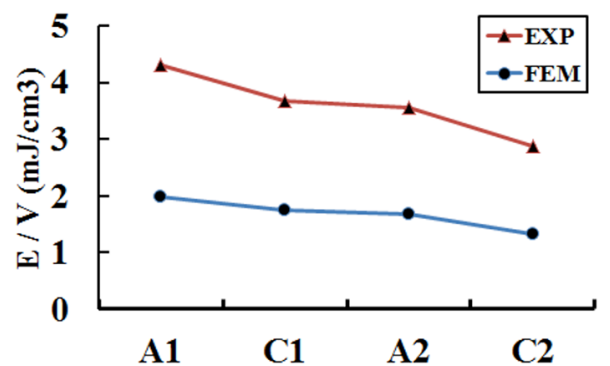

Fig. 7. Energy absorption capability $\left(\mathrm{mJ} / \mathrm{cm}^{3}\right)$ of different unit cells.

used to model the structures. The plastic hardening behavior of the structures was determined using equations (3) and (4) and data obtained from Carsi's research [38].

$$
\begin{gathered}
\sigma_{T}=\sigma_{E}\left(1+\varepsilon_{E}\right), \\
\varepsilon_{T}=\ln \left(1+\varepsilon_{E}\right)-\frac{\sigma_{T}}{E},
\end{gathered}
$$

where $\sigma_{E}$ and $\sigma_{T}$ are engineering and true stresses, respectively. Similarly, $\varepsilon_{E}$ and $\varepsilon_{T}$ are engineering and true plastic strains.

Quasi-static and dynamic loading were conducted for all structures. The quasi-static simulations were performed under the conditions described in the first paragraph of Section 4. In dynamic analysis, impactor was modeled by a rigid plate with a reference point. The mass and moment of inertia of the impactor were defined in the reference point to compare the specific energy absorption (SEA) of different structures. In order to compare the energy absorption of different structures in quasi-static analysis, the compression of each structure should be proportional to the height of the structure. Also, for dynamic loading, the mass of impactor should be proportional to the mass of the structure. In here, the mass of the impactor is the same with that of cellular auxetic structures. The velocity of the impactor is the same for all dynamic analyses with the value of $5 \mathrm{~m} / \mathrm{s}$. Surface-to-surface contact was used for contact between the rigid plates and top or bottom of the structures. Self-contact was also used for the structure itself. Explicit dynamic solver was used for simulation.

\section{Results and discussion}

\subsection{Energy absorption performance of auxetic and conventional unit cells}

Energy absorption capability of the conventional and auxetic unit cells achieved from experiments and FE simulations have been plotted and compared in Figure 7. Based on Table 1, this comparison has been carried out between unit cells A1, A2 and C1, C2 which are auxetic and conventional unit cells, respectively.

By referring to Figure 7 , it is noteworthy that the FE results are always slightly lower than the experimental results. This common discrepancy between these two results may happen due to the elastic modeling of the $\mathrm{FE}$ simulations in which, the effect of hardening behavior has been neglected. Nevertheless, the FE and experimental results indicate a good agreement. Concerning the energy absorption capability, it is obvious that the unit cells A1 and $\mathrm{A} 2$ are advantageous over the other configurations in $\mathrm{C} 1$ and $\mathrm{C} 2$. The main reason for increasing energy absorption capacity may attribute to the changing the shape of unit cells from conventional to auxetic. This structure variation leads to obtain the NPR. Previous most cited studies $[2,39,40]$ showed that in parallel to increasing in NPR, the indentation resistance and toughness are enhanced remarkably. Thus, it is evident that the auxetic unit cells have better ability to absorb energy when subjected to the uniaxial loading.

\subsection{Energy absorption performance of cellular auxetic structures}

From Section 5.1, concerning the energy absorption capacity, it is obvious that the auxetic unit cells are more advantageous compared to their conventional configurations. Therefore, some auxetic unit cells presented in Table 1 were selected to extend for providing a cellular auxetic structure. Different geometrical specification and FE samples of these cellular auxetic structures have been shown in Table 2. Also, a simple code was assigned to each structure which can be observed in the last column of this table.

Figure 8 shows the deformation modes of different cellular auxetic structures numerically. In addition, load-displacement curves of the samples achieved from the FE simulations have been plotted and compared in Figure 9. There are several indicators to estimate the energy absorption capability of a structure during the collapse. In present study, peak force $\left(P_{\max }\right)$, energy absorption (EA) and specific energy absorption (SEA) are the indicators which are employed for measuring the energy absorption capability [2]. The $P_{\max }$ determines the load required to commence collapse and to initiate the energy absorption process. Energy absorption (EA) is a criterion to demonstrate the stable limit of a structure and assist to compare among different structures. During uniaxial compression, the EA is calculated via equation (5):

$$
E A(d)=\int_{0}^{d} F(\delta) d \delta
$$

where $d$ and $\delta$ are the crushing distance and displacement respectively and $F$ denotes the crushing force. The absorbed energy per unit mass of a structure $(\mathrm{m})$ which is called SEA is determined as equation (6):

$$
S E A=\frac{E A}{m}
$$

The values of all energy absorption indictors utilized in this research are tabulated in Table 3. Also, for better comparison, the FE results of EA and SEA for both quasistatic and dynamic loadings are displayed in Figure 10. 
Table 2. Different geometrical specification and FE samples of cellular auxetic structures.

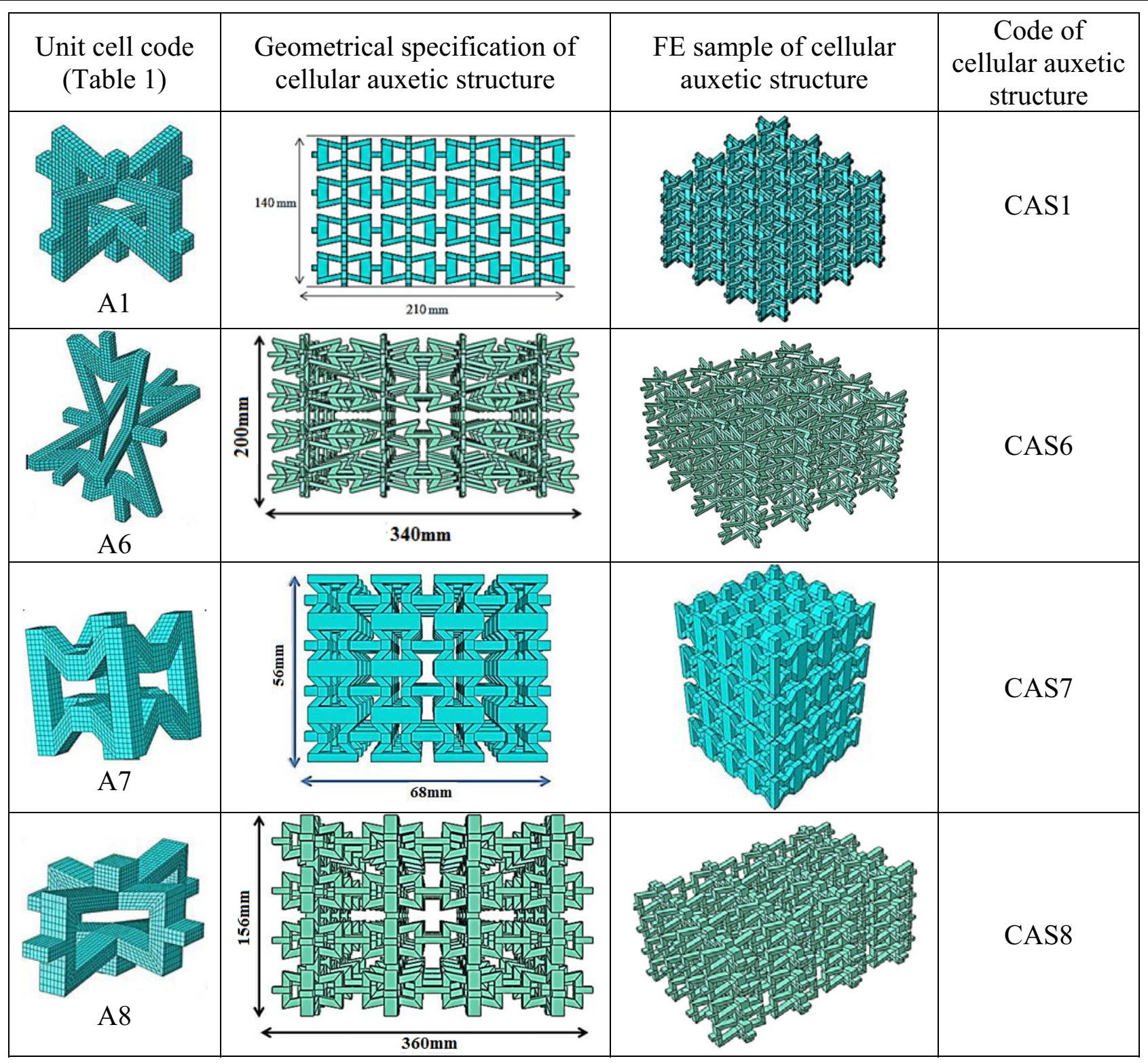

Impact resistance of the structures of the structures is defined as the average value of the force transmitted to the foundation during the impact. This parameter is shown in Figure 11.

From Figure 9 and Table 3 , the initial $P_{\max }$ of all strauctures for dynamic loading are much more than those of quasi-static loading. This can be justified by tracing the impactor location in dynamic loading state. In fact, the load is dropped because the impactor is separated from the structures.

Table 3 and Figure 9a indicate that for CAS1, the first $P_{\max }$ was around $200 \mathrm{kN}$. After very small drop, the strain hardening was happened until around $20 \mathrm{~mm}$ of crush lenght. At this specific point, the load increases dramatically due to increasing the cross section of the structure, resulting in second $P_{\max }$ at $25 \mathrm{~mm}$. Then after, the degradation of material is occurred. Figure $9 \mathrm{~b}$ shows that the first $P_{\max }$ was around $250 \mathrm{kN}$, responding to $0.1 \mathrm{~mm}$ and approximately this number was remained at the certain level until $2.5 \mathrm{~mm}$. From Figure $9 \mathrm{c}$ and Table 3 , for CAS6, the $P_{\max }$ is $300 \mathrm{kN}$. At around $34 \mathrm{~mm}$ displacement, the load increases dramatically due to increasing the cross section of the structure. The second peak load was happened late compared to the previous structure. The reason is the more space between the cells. Likewise, in Figure 9d, load drop can be observed. This means that compared with the CAS1, this structure is weaker for dynamic loading. Based on Figure 9e and Table 3, for CAS7, the first $P_{\max }$ was happened quite late, at around $4 \mathrm{~mm}$. The reason is the geometry of the structure and the space between the unit cells. The effect of this type of geometry also can be observed in Figure 9d in which, the 
(a) CAS1: Quasi-static loading

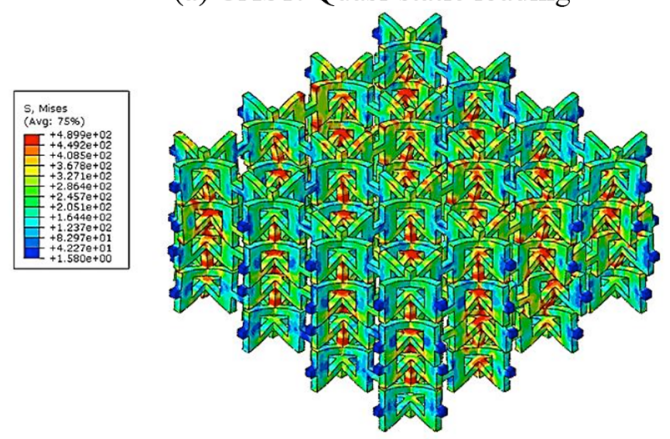

(c) CAS6: Quasi-static loading

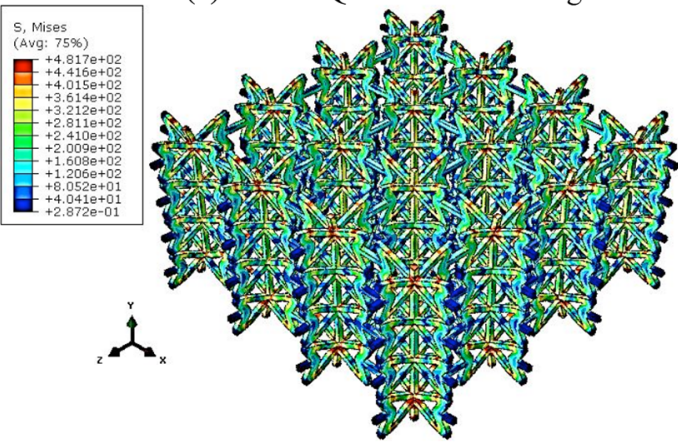

(e) CAS7: Quasi-static loading

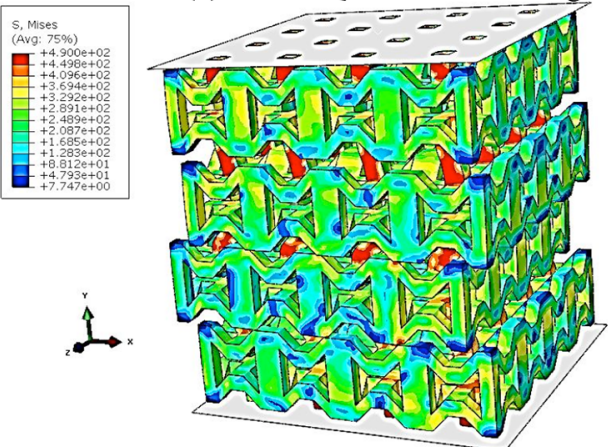

(g) CAS8: Quasi-static loading

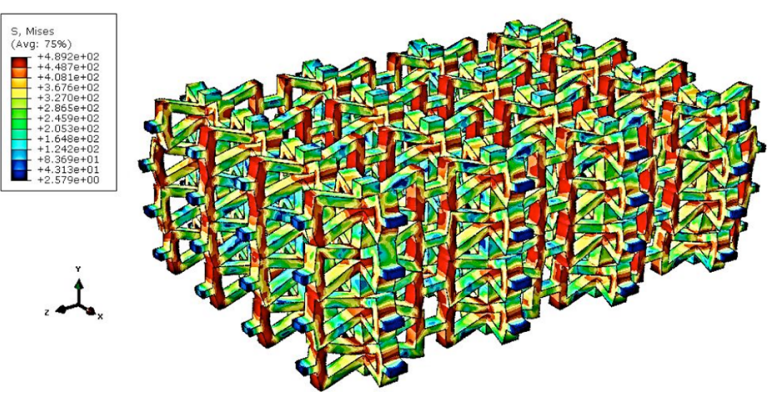

(b) CAS1: Dynamic loading

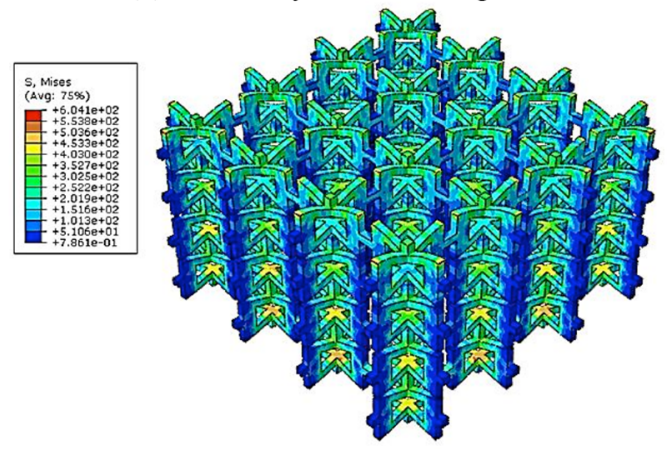

(d) CAS6: Dynamic loading
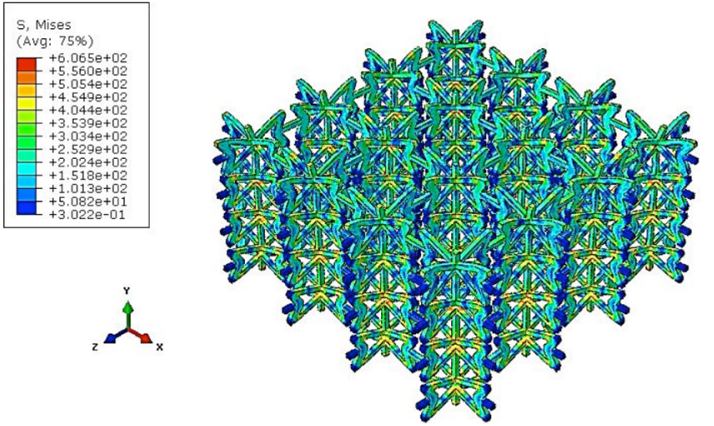

(f) CAS7: Dynamic loading
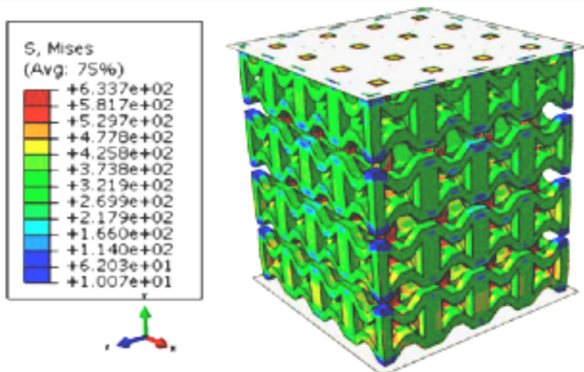

(h) CAS8: Dynamic loading
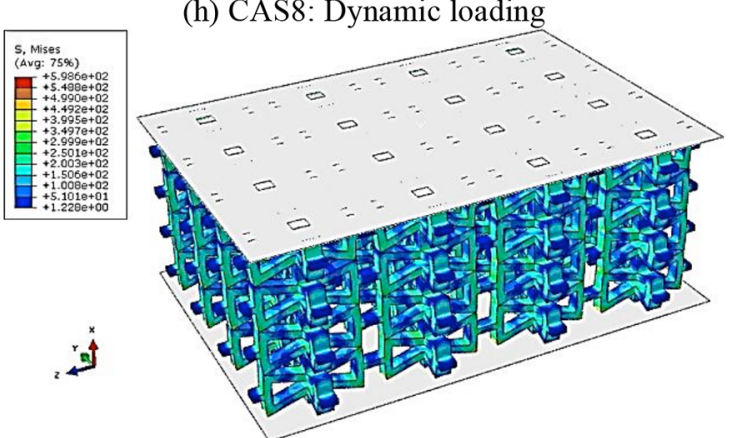

Fig. 8. Deformation modes of different structures under quasi-static and dynamic loadings.

$P_{\max }$ load is dropped suddenly. By referring to the Figures $9 \mathrm{a}$ and $9 \mathrm{~b}$ and comparing with Figures $9 \mathrm{~g}$ and $9 \mathrm{~h}$, it can be realized that, since the geometry of CAS8 is somehow similar to CAS1, their loaddisplacement curves are similar. Figure $9 \mathrm{~g}$ indicates that for CAS8, the first $P_{\max }$ was around $300 \mathrm{kN}$. For dynamic loading, this value is $600 \mathrm{kN}$ as shown in Figure $9 \mathrm{~h}$.
From Figure 10a, concerning the energy absorption capacity, it is obvious that the CAS8 and CAS1 are advantageous over the other configurations cellular auxetic structures presented in this study for both quasi-static and dynamic loadings. It can be attributed to the geometry of unit cells that these structures made by repeating them in the space. Also, the empty spaces between the unit cells in 
(a) CAS1: Quasi-static loading

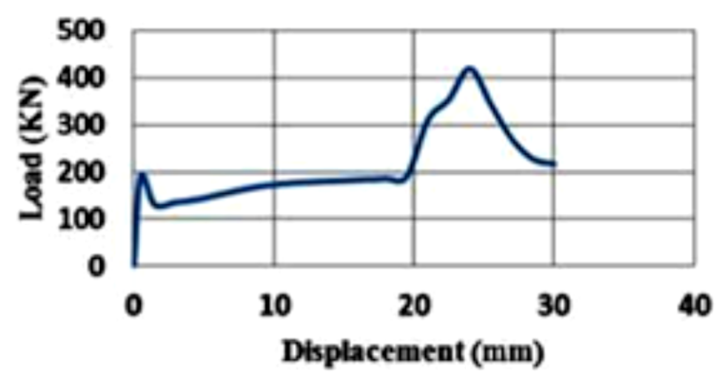

(c) CAS6: Quasi-static loading

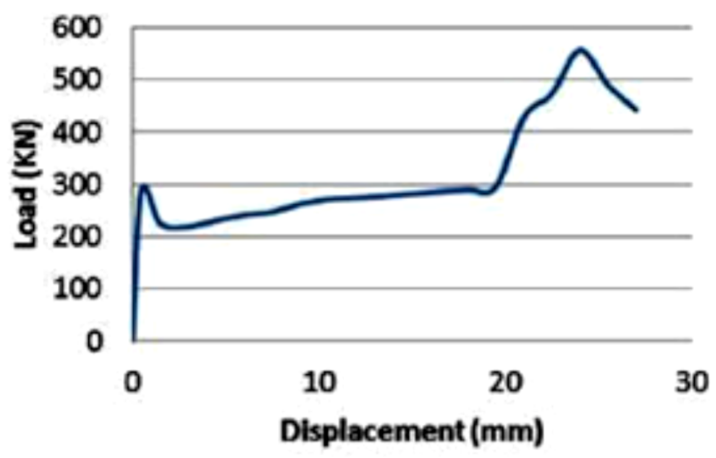

(e) CAS7: Quasi-static loading

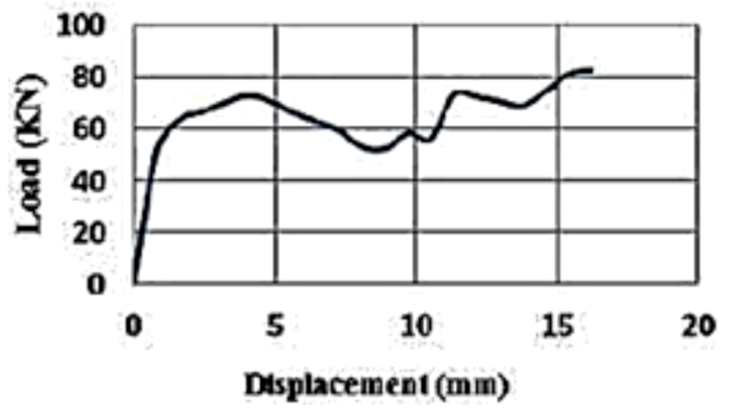

(g) CAS8: Quasi-static loading

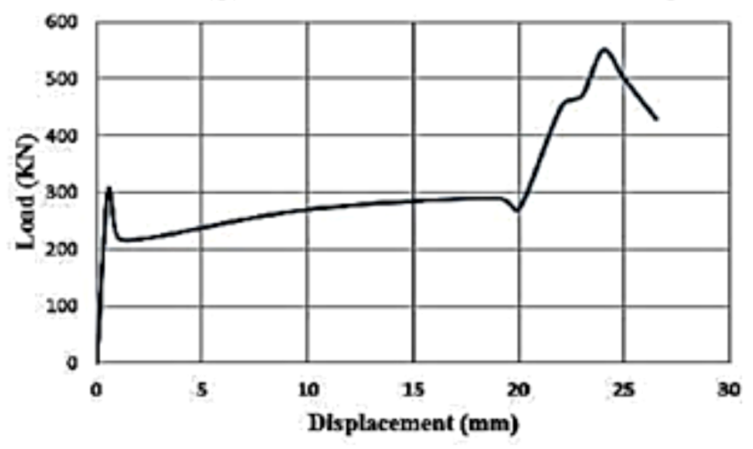

(b) CAS1: Dynamic loading

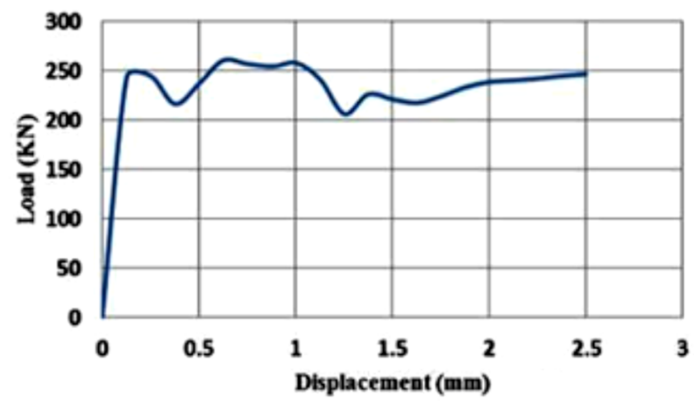

(d) CAS6: Dynamic loading

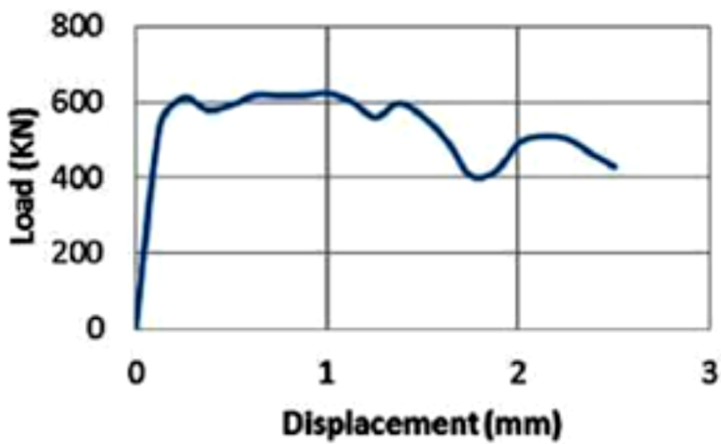

(f) CAS7: Dynamic loading

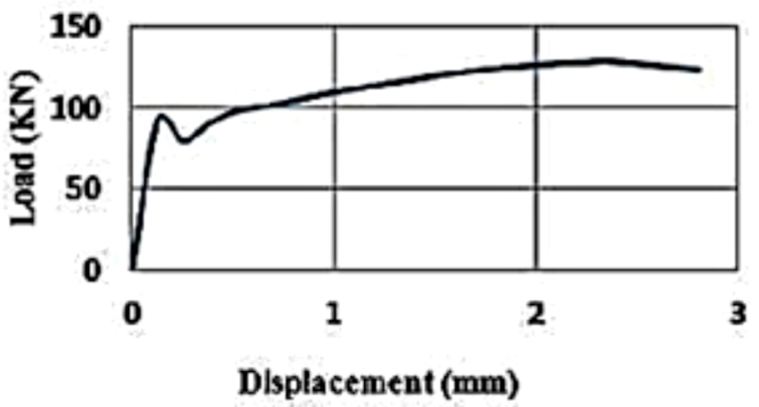

(h) CAS8: Dynamic loading

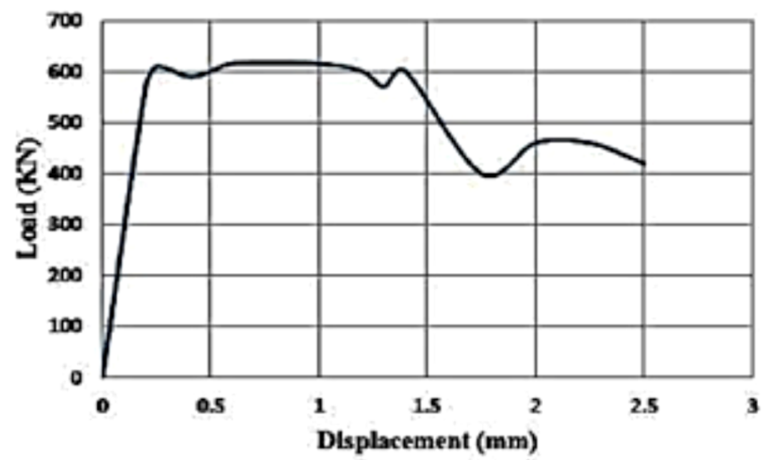

Fig. 9. Numerical load-displacement curves for different structures.

these structures are lower than the those in the CAS6 and CAS7. As a result, the structure becomes more dense causing to enhance the EA capacity.

In Figure 10b, it is noted that the SEA of CAS1 is greater than the other configurations. It can also be highlighted that the SEA of CAS7 is closed to CAS1 and
CAS8, while the EA of CAS1 was lower than that of CAS6 and CAS8, i.e., considering the mass of structure, the more absorbed energy was obtained for a given deformation. Also, it can be seen from Figure 11 that impact resistance of CAS7 and CAS6 are the greatest and lowest values among the configurations, respectively. 
Table 3. FE results from quasi-static and dynamic compressed structures.

\begin{tabular}{|c|c|c|c|c|c|c|c|}
\hline \multirow[t]{2}{*}{ Samples } & \multirow[t]{2}{*}{ Mass (kg) } & \multicolumn{3}{|c|}{ Quasi-static loading } & \multicolumn{3}{|c|}{ Dynamic loading } \\
\hline & & $P_{1 \max }(\mathrm{kN})$ & $\mathrm{EA}(\mathrm{kJ})$ & SEA $(\mathrm{kJ} / \mathrm{kg})$ & $P_{1 \max }(\mathrm{kN})$ & $\mathrm{EA}(\mathrm{kJ})$ & $\operatorname{SEA}(\mathrm{kJ} / \mathrm{kg})$ \\
\hline CAS1 & 3.83 & 217 & 2.943 & 0.774 & 247 & 0.596 & 0.134 \\
\hline CAS6 & 8.60 & 314 & 0.342 & 0.039 & 624 & 0.214 & 0.024 \\
\hline CAS7 & 0.52 & 69.5 & 0.367 & 0.571 & 95 & 0.286 & 0.427 \\
\hline CAS8 & 10.41 & 298 & 6.931 & 0.721 & 596 & 1.283 & 0.124 \\
\hline
\end{tabular}

(a)

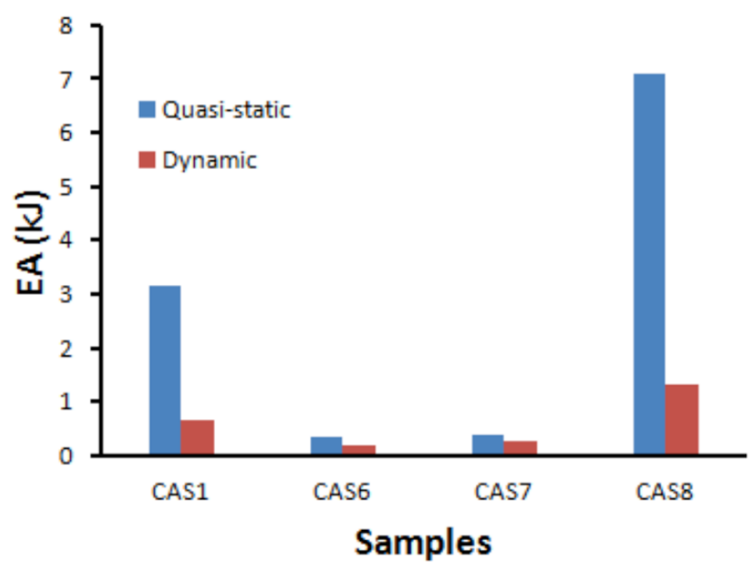

(b)

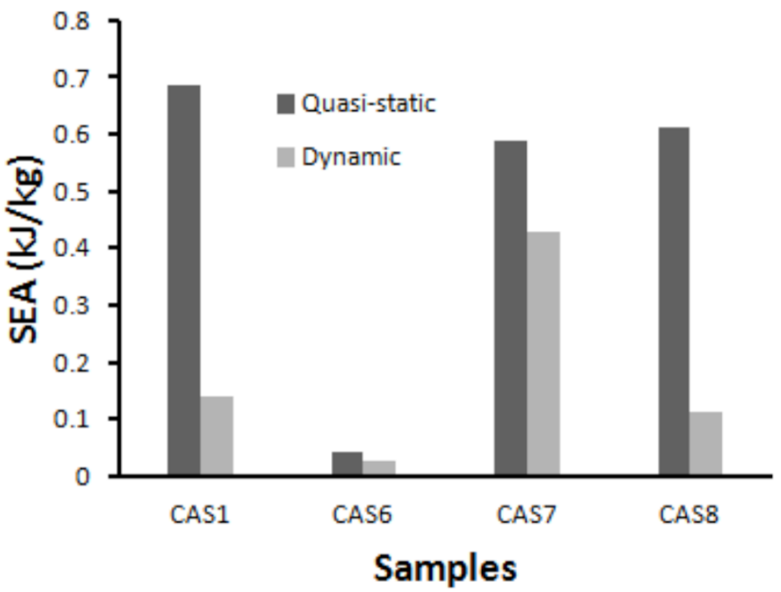

Fig. 10. Comparison between the EA and SEA of structures, a: quasi-static; b: dynamic loading.

Pav (kN)

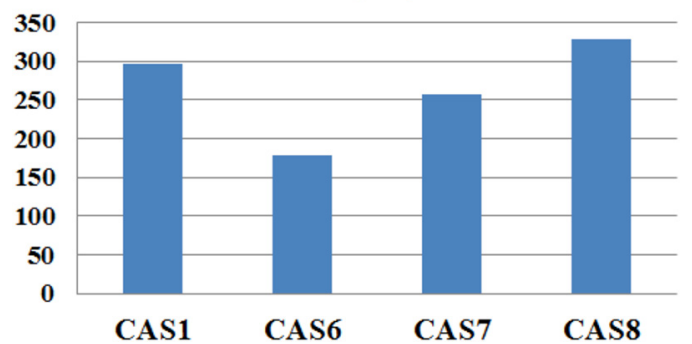

Fig. 11. Comparison between impact resistance of different auxetic structures for dynamic loading.

\section{Conclusions}

In this paper, the energy absorption of different cellular auxetic structures namely CAS1, CAS6, CAS7 and CAS8 under quasi-static and dynamic loadings condition was numerically studied. Prior to this part, the effect of using conventional and auxetic unit cells for providing the mentioned energy absorber structures was experimentally and numerically investigated. It shows a good agreement between the experiment results and simulation outcomes. A comparison between the results showed that the auxetic unit cells have greater EA in comparison with the conventional ones. So, these types of unit cells were selected to provide the energy absorber structures. In the case of cellular auxetic structures, comparison between the results revealed that the
CAS8 has greater EA in the other structures. On the other hand, when SEA is considered, the CAS7 shows good application in the area of energy absorption because it is more light weight compared to the other structures. This study has focused on the effect of using auxetic unit cells to make the energy absorber structures. Parametric study of geometrical specifications of auxetic unit cells for evaluation of energy absorption capability further can be implemented in the future work. The present outcomes of this paper may be of high interest to those engineers who intend to utilize the auxetic foam in their impact resistance design.

\section{References}

[1] Y. Prawoto, Seeing auxetic materials from the mechanics point of view: A structural review on the negative Poisson's ratio, Comput. Mater. Sci. 58 (2012) 140-153

[2] S. Mohsenizadeh, R. Alipour, M.S. Rad, A.F. Nejad, Z. Ahmad, Crashworthiness assessment of auxetic foam-filled tube under quasi-static axial loading, Mater. Des. 88 (2015) $258-268$

[3] F. Najarian, R. Alipour, M.S. Rad, A.F. Nejad, A. Razavykia, Multi-objective optimization of converting process of auxetic foam using three different statistical methods, Measurement 119 (2018) 108-116

[4] K.E. Evans, A. Alderson, Auxetic materials: Functional materials and structures from lateral thinking!, Adv. Mater. 12 (2000) 617-628 
[5] R. Critchley, I. Corni, J.A. Wharton, F.C. Walsh, R.J. Wood, K.R. Stokes, A review of the manufacture, mechanical properties and potential applications of auxetic foams, Phys. Status Solidi (b), 250 (2013) 1963-1982

[6] W. Yang, Z.-M. Li, W. Shi, B.-H. Xie, M.-B. Yang, Review on auxetic materials, J. Mater. Sci. 39 (2004) 3269-3279

[7] S. Mohsenizadeh, R. Alipour, Z. Ahmad, A. Alias, Influence of auxetic foam in quasi-static axial crushing, Int. J. Mater. Res. 107 (2016) 916-924

[8] S. Mohsenizadeh, R. Alipour, A.F. Nejad, M.S. Rad, Z. Ahmad, Experimental investigation on energy absorption of auxetic foam-filled thin-walled square tubes under quasistatic loading, Procedia Manuf. 2 (2015) 331-336

[9] T. Ting, Very large Poisson's ratio with a bounded transverse strain in anisotropic elastic materials, J. Elast. 77 (2004) 163-176

[10] R. Lakes, Experimental microelasticity of two porous solids, Int. J. Solids Struct. 22 (1986) 55-63

[11] M. Bianchi, F. Scarpa, C. Smith, Shape memory behaviour in auxetic foams: Mechanical properties, Acta Mater. 58 (2010) 858-865

[12] J.N. Grima, D. Attard, R. Gatt, R.N. Cassar, A novel process for the manufacture of auxetic foams and for their reconversion to conventional form, Adv. Eng. Mater. 11 (2009) 533-535

[13] M. Bianchi, S. Frontoni, F. Scarpa, C. Smith, Density change during the manufacturing process of PU-PE open cell auxetic foams, Phys. Status Solidi (b), 248 (2011) 30-38

[14] S. Shilko, D. Konyok, Numerical and experimental study of auxetic closed-cell foams, Comput. Methods Sci. Technol. 10 (2004) 197-202

[15] M. Bianchi, F.L. Scarpa, C.W. Smith, Stiffness and energy dissipation in polyurethane auxetic foams, J. Mater. Sci. 43 (2008) 5851-5860

[16] M. Bianchi, F. Scarpa, C. Smith, G.R. Whittell, Physical and thermal effects on the shape memory behaviour of auxetic open cell foams, J. Mater. Sci. 45 (2010) 341

[17] Y.C. Wang, R. Lakes, A. Butenhoff, Influence of cell size on re-entrant transformation of negative Poisson's ratio reticulated polyurethane foams, Cell. Polym. 20 (2001) 373-385

[18] J.R. Wright, M.K. Burns, E. James, M.R. Sloan, K.E. Evans, On the design and characterisation of low-stiffness auxetic yarns and fabrics, Text. Res. J. 82 (2012) 645-654

[19] K. Evans, J. Donoghue, K. Alderson, The design, matching and manufacture of auxetic carbon fibre laminates, J. Compos. Mater. 38 95-106 (2004)

[20] K. Alderson, R. Webber, A. Kettle, K. Evans, Novel fabrication route for auxetic polyethylene. Part 1. Processing and microstructure, Polym. Eng. Sci. 45 (2005) 568-578

[21] J.N. Grima, R. Gatt, N. Ravirala, A. Alderson, K.E. Evans, Negative Poisson's ratios in cellular foam materials, Mater. Sci. Eng. A, 423 (2006) 214-218

[22] R. Blumenfeld, S.F. Edwards, Theory of strains in auxetic materials, J. Supercond. Nov. Magn. 25, 565-571 (2012)
[23] F. Scarpa, L. Ciffo, J. Yates, Dynamic properties of high structural integrity auxetic open cell foam, Smart Mater. Struct. 13 (2003) 49

[24] L. Yang, O. Harrysson, H. West, D. Cormier, Design and characterization of orthotropic re-entrant auxetic structures made via EBM using Ti6Al4V and pure copper, in: International Solid Freeform Fabrication Symposium, 2011

[25] F. Dos Reis, J. Ganghoffer, Equivalent mechanical properties of auxetic lattices from discrete homogenization, Computat. Mater. Sci. 51 (2012) 314-321

[26] H. Wang, Z. Lu, Z. Yang, X. Li, A novel re-entrant auxetic honeycomb with enhanced in-plane impact resistance, Compos. Struct. 208 (2019) 758-770

[27] X. Zhao, Q. Gao, L. Wang, Q. Yu, Z. Ma, Dynamic crushing of double-arrowed auxetic structure under impact loading, Mater. Des. 160 (2018) 527-537

[28] L. Hu, M.Z. Zhou, H. Deng, Dynamic indentation of auxetic and non-auxetic honeycombs under large deformation, Compos. Struct. 207 (2019) 323-330

[29] S. Reid, C. Peng, Dynamic uniaxial crushing of wood, Int. J. Impact Eng. 19 (1997) 531-570

[30] D. Ruan, G. Lu, B. Wang, T.X. Yu, In-plane dynamic crushing of honeycombs-A finite element study, Int. J. Impact Eng. 28 (2003) 161-182

[31] X.C. Zhang, H.M. Ding, L.Q. An, X.L. Wang, Numerical investigation on dynamic crushing behavior of auxetic honeycombs with various cell-wall angles, Adv. Mech. Eng. 7 (2014) 1-12

[32] L. Hu, F. You, T. Yu, Effect of cell-wall angle on the in-plane crushing behaviour of hexagonal honeycombs, Mater. Des. 46 (2013) 511-523

[33] Z. Zou, S. Reid, P. Tan, S. Li, J. Harrigan, Dynamic crushing of honeycombs and features of shock fronts, Int. J. Impact Eng. 36 (2009) 165-176

[34] L. Hu, T. Yu, Dynamic crushing strength of hexagonal honeycombs, Int. J. Impact Eng. 37 (2010) 467-474

[35] L. Hu, T. Yu, Mechanical behavior of hexagonal honeycombs under low-velocity impact-theory and simulations, Int. J. Solids Struct. 50 (2013) 3152-3165

[36] Z.X. Lu, Q. Liu, Z.Y. Yang, Predictions of Young's modulus and negative Poisson's ratio of auxetic foams, Phys. Status Solidi (b), 248 (2011) 167-174

[37] M.S. Rad, Y. Prawoto, Z. Ahmad, Analytical solution and finite element approach to the $3 \mathrm{D}$ re-entrant structures of auxetic materials, Mech. Mater. 74 (2014) 76-87

[38] M. Carsí, A. Fernández-Vicente, O.A. Ruano, O. Sherby, Processing, microstructure, strength, and ductility relationships in ultrahigh carbon steel assessed by high strain rate torsion testing, Mater. Sci. Technol. 15 (1999) 1087-1095

[39] J.N. Grima, R. Caruana-Gauci, D. Attard, R. Gatt, Threedimensional cellular structures with negative Poisson's ratio and negative compressibility properties, Proc. R. Soc. A 468 (2012) 3121-3138

[40] F. Scarpa, J. Yates, L. Ciffo, S. Patsias, Dynamic crushing of auxetic open-cell polyurethane foam, Proc. Insti. Mech. Eng., Part C: J. Mech. Eng. Sci. 216 (2002) 1153-1156

Cite this article as: M. Shokri Rad, H. Hatami, R. Alipouri, A. Farokhi Nejad, F. Omidinasab, Determination of energy absorption in different cellular auxetic structures, Mechanics \& Industry 20, 302 (2019) 